\title{
Tumor lysis syndrome associated with carboplatin and paclitaxel in a woman with recurrent endometrial cancer
}

\author{
Heidi Godoy, Joshua P. Kesterson, and Shashikant Lele \\ Division of Gynecologic Oncology, Roswell Park Cancer Institute, Buffalo, NY, USA
}

\section{Keywords}

Recurrent endometrial cancer; Tumor lysis syndrome

\begin{abstract}
Tumor lysis syndrome (TLS) consists of a constellation of metabolic derangements including hyperuricemia, hyperkalemia, hyperphosphatemia, uremia, and hypocalcemia, usually triggered by cytotoxic chemotherapy. It results from the lysis of malignant cells with a resultant immense and rapid release of cellular contents from lysed neoplastic cells. TLS is rare in solid tumors [1]. Here we describe a patient who developed TLS after carboplatin and paclitaxel for recurrent endometrial cancer.
\end{abstract}

A 60-year-old woman with a history of FIGO stage IIB uterine cancer receiving carboplatin and paclitaxel for recurrent endometrial cancer presented to the emergency room with shortness of breath. The patient had received carboplatin and paclitaxel 4 days previously. She admitted to a several day history of shortness of breath, weakness, and fatigue. Upon arrival she was hypotensive and tachycardic. Physical examination showed that the woman was in discomfort with decreased breath sounds at the lung bases and a diffusely tender abdomen. Laboratory evaluation revealed several abnormalities (Table 1). A CT scan showed bilateral atelectasis with consolidation at the lung bases and multiple mesenteric and peritoneal implants. The patient was given empiric antibiotics and intravenous hydration for suspected septicemia. Repeat lab values revealed worsening metabolic abnormalities consistent with TLS (Table 1). Despite aggressive interventions including dialysis and attempted correction of her metabolic and electrolyte derangements, her condition deteriorated and she died on hospital day 2.

TLS is exceedingly rare in solid malignancies. In a review by Baeksgaard and Sørensen [1], they noted only 45 cases of TLS had been reported in patients with solid tumors. We are aware of only 5 reported cases occurring in gynecologic malignancies [1-3].

TLS results from the lysis of tumor cells with the release of their intracellular contents into the bloodstream. This leads to metabolic derangements including hyperuricemia, hyperphosphatemia, hyperkalemia, hypocalcemia, and elevated lactate dehydrogenase

\footnotetext{
*Corresponding author. Division of Gynecologic Oncology, Roswell Park Cancer Institute, Elm and Carlton Streets, Buffalo, NY 14263, USA. Tel.: +1 716845 5776; fax: +1 716845 7608. Shashi.Lele@ roswellpark.org (S. Lele).

Conflict of interest

The authors have no conflicts of interest to report.
} 
(LDH). These electrolyte abnormalities, if uncorrected, can lead to cardiovascular, renal, and neuromuscular compromise. Clinically, patients may present with acute renal failure, nausea, vomiting, diarrhea, anorexia, hematuria, cardiac arrhythmias, seizures, hypotension, and neuromuscular symptoms, including muscle cramps and paresthesia [4]. Our patient presented with shortness of breath, fatigue, and malaise. Laboratory evaluation revealed profound electrolyte disturbances. She required hemodialysis secondary to renal failure and metabolic derangements. Unfortunately, this patient's condition deteriorated rapidly and she died 2 days after presentation and aggressive management. The mortality rate of TLS associated with solid tumors is 1 in 3 [1].

The optimal management of TLS is its prevention. Effective prevention involves recognition of those at risk with close monitoring and frequent assessment for electrolyte abnormalities. Patients at risk should receive aggressive hydration and diuresis with administration of an antihyperuricemic agent (e.g. allopurinol, rasburicase) [4].

We report a case of TLS in a patient receiving carboplatin and paclitaxel for recurrent endometrial cancer. Despite the infrequency of its occurrence in the gynecologic oncology setting, clinicians need to be aware of those at risk, its clinical and laboratory presentation, treatment, and prophylaxis because of its potential lethality.

\section{References}

[1]. Baeksgaard L, Sorensen JB. Acute tumor lysis syndrome in solid tumors. Cancer Chemother Pharmacol. 2003; 51(3):187-92. [PubMed: 12655435]

[2]. Chan JK, Lin SS, McMeekin DS, Berman ML. Patients with malignancy requiring urgent therapy: Case 3. Tumor lysis syndrome associated with chemotherapy in ovarian cancer. J Clin Oncol. 2005; 23(27):6794-5. [PubMed: 16170188]

[3]. Yahata T, Nishikawa N, Aoki Y, Tanaka K. Tumor lysis syndrome associated with weekly paclitaxel treatment in a case with ovarian cancer. Gynecol Oncol. 2006; 103(2):752-4. [PubMed: 16857250]

[4]. Rampello E, Fricia T, Malaguarnera M. The management of tumor lysis syndrome. Nat Clin Pract Oncol. 2006; 3(8):438-47. [PubMed: 16894389] 


\section{Table 1}

Laboratory values before and after carboplatin and paclitaxel.

\begin{tabular}{lccc}
\hline Parameter & $\begin{array}{l}\text { Before } \\
\text { chemotherapy }\end{array}$ & $\begin{array}{l}\text { After chemotherapy } \\
\text { (day 4) }\end{array}$ & $\begin{array}{l}\text { After chemotherapy } \\
\text { (day 5) }\end{array}$ \\
\hline Leukocytes, $\times 10^{3} / \mathrm{mm}^{3}$ & 9.9 & 2.27 & 0.33 \\
Hemoglobin, g/dL & 10.5 & 10.4 & 10 \\
Potassium, mEq/L & 4.4 & 7.7 & 5.7 \\
Phosphate, mg/dL & & 11.4 & 7.5 \\
BUN, mg/dL & 25 & 94 & 97 \\
Creatinine, mg/dL & 1.0 & 3.2 & 2.6 \\
Uric acid, mg/dL & & 17.1 & 15.4 \\
AST, IU/L & & 3058 & 6578 \\
ALT, IU/L & & 1166 & 962 \\
LDH, IU/L & & 29380 & 68264 \\
\hline
\end{tabular}

Abbreviations: BUN, blood urea nitrogen; AST, aspartate aminotransferase; ALT, alanine aminotransferase; LDH, lactate dehydrogenase. 